\title{
Normalization of impaired emotion inhibition in bipolar disorder mediated by cholinergic neurotransmission in the cingulate cortex
}

Leila Nabulsi (iD ${ }^{1,2 凶}$, Jennifer Farrell ${ }^{1}$, Genevieve McPhilemy ${ }^{1}$, Liam Kilmartin ${ }^{3}$, Maria R. Dauvermann ${ }^{1,4}$, Theophilus N. Akudjedu (iD ${ }^{1,5}$, Pablo Najt ${ }^{1}$, Srinath Ambati ${ }^{1}$, Fiona M. Martyn (iD) ${ }^{1}$, James McLoughlin ${ }^{1}$, Michael Gill (iD ${ }^{6}$, James Meaney ${ }^{6}$, Derek Morris (iD) ${ }^{1}$, Thomas Frodl (iD ${ }^{6,7}$, Colm McDonald (iD) ${ }^{1}$, Brian Hallahan ${ }^{1}$ and Dara M. Cannon (iD) ${ }^{1}$

(c) The Author(s) 2022

The muscarinic-cholinergic system is involved in the pathophysiology of bipolar disorder (BD), and contributes to attention and the topdown and bottom-up cognitive and affective mechanisms of emotional processing, functionally altered in BD. Emotion processing can be assessed by the ability to inhibit a response when the content of the image is emotional. Impaired regulatory capacity of cholinergic neurotransmission conferred by reduced $\mathrm{M}_{2}$-autoreceptor availability is hypothesized to play a role in elevated salience of negative emotional distractors in euthymic BD relative to individuals with no history of mood instability. Thirty-three euthymic BD type-I (DSM-V-TR) and 50 psychiatrically-healthy controls underwent functional magnetic resonance imaging (fMRI) and an emotioninhibition paradigm before and after intravenous cholinergic challenge using the acetylcholinesterase inhibitor, physostigmine (1 mg), or placebo. Mood, accuracy, and reaction time on either recognizing or inhibiting a response associated with an image involving emotion and regional functional activation were examined for effects of cholinergic challenge physostigmine relative to placebo, prioritizing any interaction with the diagnostic group. Analyses revealed that (1) at baseline, impaired behavioral performance was associated with lower activation in the anterior cingulate cortex in BD relative to controls during emotion processing; (2) physostigmine (vs. placebo) affected behavioral performance during the inhibition of negative emotions, without altering mood, and increased activation in the posterior cingulate cortex in $\mathrm{BD}$ (vs. controls); (3) In BD, lower accuracy observed during emotion inhibition of negative emotions was remediated by physostigmine and was associated with cingulate cortex overactivation. Our findings implicate abnormal regulation of cholinergic neurotransmission in the cingulate cortices in $\mathrm{BD}$, which may mediate exaggerated emotional salience processing, a core feature of BD.

Neuropsychopharmacology (2022) 47:1643-1651; https://doi.org/10.1038/s41386-022-01268-7

\section{INTRODUCTION}

Bipolar disorder (BD) is a severe and burdensome psychiatric condition involving recurrent depressive and manic episodes and overall instability in regulating emotions [1]. The severity and burden of bipolar affective dysregulation affects the quality of life of approximately $1-3 \%$ of the world population [2]; however, the neurobiological basis leading to the illness remains unclear. Current pharmacological intervention of BD includes mood stabilizers and antipsychotics; however, the time lag in efficacy for these drugs, or inefficiency [3], makes BD treatment suboptimal resulting in a proportion of patients remaining refractory to treatment. Hence, there is a greater need for the discovery of new agents that are specific to biologically defined streams within BD. Using pharmacological neuroimaging research, it is important to identify neural biomarkers with predictive validity for therapeutic response to enhance diagnostic specificity and guide treatment decisions, to identify the different biological subtypes of BD that may exist and to address the biological heterogeneity extant within mood disorders.

Emotion processing involves the detection and evaluation of salient stimuli as well as the regulation of one's emotional (affective) response to these stimuli [4]. Dysregulated emotional responses can lead to pathological mood states. Among the array of emotion-related impairments in $\mathrm{BD}$, a hallmark of this disorder is the distorted information processing or attentional allocation toward emotional stimuli [5]. In particular, the salience of negative stimuli is proven challenging in life for those presenting with the disorder [6]. Impaired ability to inhibit a response to emotional

\footnotetext{
${ }^{1}$ Center for Neuroimaging, Cognition and Genomics (NICOG), Clinical Neuroimaging Lab, NCBES Galway Neuroscience Centre, College of Medicine, Nursing, and Health Sciences, National University of Ireland Galway, H91 TK33 Galway, Ireland. ${ }^{2}$ Imaging Genetics Center, Mark and Mary Stevens Neuroimaging \& Informatics Institute, University of Southern California, Marina del Rey, CA 90292, USA. ${ }^{3}$ College of Engineering and Informatics, National University of Ireland Galway, Galway, Ireland. ${ }^{4}$ Department of Forensic and Neurodevelopmental Sciences, Institute of Psychiatry, Psychology and Neuroscience, King's College London, London SE5 8AF, UK. Institute of Medical Imaging \& Visualisation,

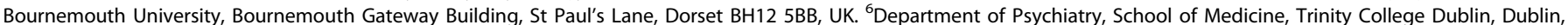
Ireland. ${ }^{7}$ Department of Psychiatry and Psychotherapy, Otto-von-Guericke-Universität Magdeburg, University Hospital Magdeburg, Magdeburg, Germany.

凶email: Inabulsi@usc.edu
}

Received: 21 September 2021 Revised: 13 December 2021 Accepted: 4 January 2022

Published online: 19 January 2022 
1644

stimuli (i.e., the emotion-inhibition condition of the functional task in this study) is one aspect of emotion regulation that may be caused by distorted perception and exaggerated responses to emotional stimuli [7]. Emotion processing can be assessed by the ability to inhibit a response when the content of the image is emotional - the emotion-inhibition condition of the fMRI task assessed herein. Although these are clinically and functionally relevant key features of manic and depressive states in $\mathrm{BD}$, and to a lesser extent of euthymia [7], the neural mechanisms that underpin these impairments are poorly understood. Relating clinically meaningful endpoints to neurotransmitter system circuits is feasible using pharmacological challenge during functional task-based MRI and has the potential to inform more precise symptom management.

Much evidence converges in implicating a molecular signature in human emotion processing, with specific neuromodulatory systems modulating task-specific cognitive states and emotions [8]. In terms of $\mathrm{BD}$, human emotion processing is relevant in the following context. The hypothesis that the brain's cholinergic neurotransmitter system could act as a modulator of depression and mania was originally proposed in the 1970s by Janowsky and colleagues in their cholinergic-adrenergic hypothesis of mania and depression [9], recently revisited [10], whereby increased and decreased central cholinergic states in the brain could contribute to low and elevated mood states, respectively. Behaviorally the cholinergic system plays major roles in attention, and the topdown and bottom-up mechanisms of emotion processing [11-13]; cognitive mechanisms known to be functionally impaired among individuals with in BD [4]. Numerous lines of evidence have since shed further light on the role of the cholinergic system in moodregulation and specifically for the muscarinic-cholinergic system in $\mathrm{BD}$, drawing upon post-mortem, genetic, pre-clinical and clinical studies [14].

Studies using adequate doses of cholinomimetic agents such as physostigmine have been shown to exacerbate depressive signs and symptoms in actively depressed subjects [15], and in euthymic individuals with BD [15-17]; as well as psychiatricallyhealthy individuals [15-18]. These effects are mediated centrally as administration of simplified analogues of physostigmine not crossing the blood-brain barrier, such as neostigmine, do not result in these depressive effects [19]. Crucially, these effects are mediated by muscarinic receptors $[20,21]$ as they are reversed by antimuscarinic agents such as biperiden [22]. Furthermore, cholinomimetic drugs, including physostigmine have been demonstrated to play a role in the reversal of mania in BD [23-27]. It is noteworthy that various monoamine transmitters may interplay with the muscarinic-cholinergic system in mechanisms of depression and mania, implying a muscarinic-cholinergic modulatory effect on mood [28]. Further evidence for cholinergic system involvement in BD comes from in vivo molecular positron emission tomography where Cannon and colleagues [29] showed that the muscarinic-cholinergic type-2 receptor $\left(M_{2}\right)$, an inhibitory or autoreceptor, is less available in the limbic system and particularly in the cingulate cortices in $\mathrm{BD}$ relative to major depressive disorder or controls [29]. In a follow-up study, Cannon and colleagues [30] suggested that changes in $M_{2}$-receptor availability may be led by a variation in the gene (CHRM2) coding for the $\mathrm{M}_{2}$-receptor ( $T$ T genotype of SNP rs324650). Cannon and colleagues [30] in their study of BD using [18 F]FP-TZTP radioligand, sensitive to intersynaptic concentrations of $A C h$, suggest that the observations of reduced PET binding in BD subjects may be attributable to differences either in M2-receptor density or affinity, or intrasynaptic ACh concentrations. In recent large-scale GWAS studies, several polymorphisms of the CHRM2 gene have been associated with features of cognitive decline in healthy controls [31], though not rs324650 specifically. Although large-scale GWAS studies have not yet identified cholinergic genes as prominent risk factor for $\mathrm{BD}$, evidence for cholinergic genes involvement in BD illness have been derived from small scale studies [6].

BD is associated with structural and functional abnormalities that overlap with regions involved in ventromedial and ventrolateral routes to emotional control $[1,32,33]$. Studies focusing on functional MRI ( $\mathrm{FMRI}$ ) activation in euthymic BD in relation to emotion processing reported increased activation in frontolimbic regions (including amygdala, nucleus accumbens and prefrontal cortex $[34,35])$ and decreased activation in the basal ganglia (caudate, putamen and globus pallidum [36, 37]) and in the inferior and middle frontal cortices [36-39]. These areas overlap with the ventral-dorsal systems thought to underpin the processing of emotion and participate in circuits responsible for attributing and perceiving the salience of emotional information $[40,41]$. These areas are rich in cholinergic projections [42] and overlap anatomically with regions of previously implicated low muscarinic-cholinergic receptor distribution volume in BD [29]. Taken together with the robust pharmacological evidence for mood effect of cholinomimetics agents reviewed earlier it is clear that the muscarinic-cholinergic system is implicated in aspects of BD illness.

In a randomized, double-blind placebo-controlled study using the acetylcholinesterase inhibitor physostigmine during an emotion-inhibition fMRI paradigm, we aimed to demonstrate muscarinic-cholinergic involvement in clinically relevant behavioural features of $\mathrm{BD}$ relative to a control group. We examined behavioural performance (accuracy and reaction time) and related neural abnormalities during processes of emotion recognition and emotion inhibition. Our hypothesis is that subjects with BD will display reduced capacity for inhibitory regulation of muscariniccholinergic neurotransmission upon stimulation of the system with physostigmine relative to placebo and control groups. Further, that physostigmine would impact behavioural performance (accuracy and reaction time) in terms of inhibition of negative emotional stimuli, and that the effects observed in BD will exceed the expected effects in healthy controls. We did not anticipate that physostigmine would significantly alter mood at the given dosage of $1 \mathrm{mg}$, considering worsening of mood had been observed only at higher dosages in both euthymic and actively depressed BD subjects $[15,16]$. The expected impact of this research is to inform on the contribution of the muscariniccholinergic neurotransmitter system to BD; and highlight the relevance of developing therapeutics targeting this system to ameliorate core emotional clinically relevant features of BD.

\section{MATERIALS AND METHODS \\ Emotion recognition and inhibition task}

In $\mathrm{BD}$, there is evidence that cognitive performance is impaired when there are emotional stimuli in the environment. Participants were trained on the task prior to MRI scanning; participants were first asked to stare at a fixation cross, then asked to process visual stimuli with different emotional valence, followed by a question referring either to the emotional valence of the picture (the emotion-recognition trial) or to the image orientation (the emotion-inhibition trial). The emotion-recognition trial involved participants to focus on the emotional content of the picture, namely if the image had positive, negative or no (neutral) emotional valence. The image orientation trial involved participants to focus on the physical orientation of the frame of the picture, namely if the image was in portrait (vertical) orientation or not. Participants answered 'yes' or 'no' using their dominant hand on a two-button response box (Current Design Inc, USA). Participants did not know which of the trial types they would be asked, which forced them to process both types of information (emotion and orientation), ultimately having to inhibit one type depending on the question asked. Accuracy (number of accurate hits) and reaction times (ms) were collected (Presentation Software, Neurobehavioral Systems $18.0 \mathrm{v}$, Neurobehavioral Systems, Inc., Berkeley, CA, www.neurobs.com). To remove outliers, in each fMRI task accuracy and reaction time were thresholded at the individual level. For reaction times (RT), a selective mean was calculated as the overall mean $_{\mathrm{RT}} /$ standard deviation $_{\mathrm{RT}}$ and only 
those values falling within \pm two standard deviations from the selective mean were extracted for each event type; in other words, subjects who were too fast or too slow at answering the question asked were considered outliers and thus not analyzed. Based on this RT threshold, accuracy was extracted for each event type, i.e. the number of hits for those subjects considered outliers (based on RT) were not analyzed.

\section{Study design \& pharmacological challenge}

This study was designed as a randomized double-blind placebo-controlled trial involving subjects undergoing fMRI task-based imaging (acquisition and task detailed below) before and after a pharmacological challenge of the cholinergic system. After the first fMRI task session and 30 min prior to the start of the second task session, participants underwent intravenous infusion of placebo (sodium chloride $0.9 \%$ ) or physostigmine, at an initial dose of $2 \mathrm{mg} / \mathrm{hr}$ for $10 \mathrm{~min}$ followed by $0.8 \mathrm{mg} / \mathrm{hr}$ to completion of the study resulting in a maximum possible dose of $1 \mathrm{mg}$ physostigmine [43]. Glycopyrrolate $0.2 \mathrm{mg}$ i.v. was administered before physostigmine infusion to minimize peripheral side effects of physostigmine [44]. At this dose and rate of infusion physostigmine has been demonstrated to have minimal side effects while altering behavioural performance to a detectable level [11].

\section{Study participants diagnosis \& genotype}

Participants aged between 18 and 65 years were recruited from the western regions of Ireland's Health Services via referral (outpatients) or public advertisement (patients and controls). All participants were genotyped (detailed below) for the muscarinic-cholinergic type-2 receptor gene (CHRM2) single nucleotide polymorphism (SNP) rs324650 and matched for genotype (AA, AT and TT) within diagnostic groups. Groups were matched for age within a range of $+/-5$ years. A diagnosis of $B D$ was confirmed using the Diagnostic and Statistical Manual of Mental Disorders (DSM-IVTR) [45] 40 and the Structured Clinical Interview by an experienced psychiatrist; euthymia was defined using the Hamilton Depression (HDRS$21<8$ ) and Young Mania (YMRS $<7$ ) Rating Scales at both medical screening and MRI scanning. Additionally, anxiety symptoms were assessed using the Hamilton Anxiety rating scale (HARS $<18$ ). Exclusion criteria included neurological disorders, learning disability, comorbid misuse of substance/alcohol and of axis-1 disorders, history of head injury resulting in loss of consciousness for $>5$ min or a history of oral steroid use in the previous 3 months, unstable respiratory conditions, or history of cardiovascular events. Healthy controls had no personal history of a psychiatric illness or history of first-degree relatives (DSM-IV, Non-patient edition). A licensed pharmacist reviewed drug interactions with the cholinomimetic agent used in the study prior to administration. Ethical approval for the study was granted by the University College Hospital Galway, St. James's Hospital Dublin and Mullingar CRECS, and participants gave written fully informed consent.

\section{Mood and word salience rating scale}

The Profile of Mood States (POMS) questionnaire, the Visual Analogue scale [46] (VAS) and a word salience rating scale were administered before and after MRI scanning. Data on both were missing in one BD and one control participant. The POMS consisted of 65 emotionally valent words rated on a scale of 1, 'not at all' to 5, 'extremely'. POMS scores formed four independent factors (Supplementary Table 1; Supplementary Fig. 1) using a principal component analysis, namely 'depressed' ( $r$ range:0.88-0.52), 'fatigued' $(r: 0.82-0.52)$, 'positive' (r:0.75-0.50) and 'irritable' ( $r: 0.87-0.54)$. The VAS scores were found to encompass in the POMS scores entirely in terms of variance. Additionally, subjects subjectively rated the salience of previously validated [47] 79 emotionally valenced words on a scale of 'very positive' to 'very negative'. Repeated measure Multivariate Analysis of Co-Variance (MANCOVA) with fixed factors diagnosis and challenge was carried out for group comparisons $(p<0.05)$. All statistical analyses were carried out using the Statistics Package for Social Sciences (SPSS v23, IBM, New York, USA).

\section{Emotion recognition and inhibition $\mathrm{fMRI}$ task}

An emotion-inhibition fMRI task was chosen to assess regional functional activation involved in valence assessment of emotional content $[48,49]$, consisting of 180 pseudo-randomized trials belonging to two groups, emotion-recognition and emotion-inhibition trials, and three emotional valences for a total of six event types (International Affective Picture System database, IAPS [50]). Two unique batches of images matched for the degree of salience of each valence were used before and after physostigmine or placebo administration, resulting in sixty unrepeated pictures in each valence category (mean time of fMRI task events $=1.5 \mathrm{~s}$ ). For further details on the fMRI paradigm and behavioural data acquisition refer to Supplementary Material.

We examined baseline behavioural performance (accuracy and reaction time) in BD relative to controls on placebo (MANCOVA, fixed factor diagnosis, covarying for age and gender, $p<0.05)$; and after physostigmine administration. Comparisons included the interaction between drug treatment, physostigmine or placebo, and diagnostic groups; and the effect of drug treatment within each diagnostic group (repeated measure MANCOVA, fixed factors diagnosis and challenge, covarying for age and gender; $p<0.05)$. Post-hoc investigations were carried out on the significant group differences (one-way ANOVA; $p_{\text {FDR-corrected }}<0.05$;).

\section{Image acquisition \& processing}

MRI scanning was performed at the Welcome Trust Health Research Board National Centre for Advanced Medical Imaging (CAMI) at St. James's Hospital Dublin, Ireland, using a 3 Tesla Achieva scanner (Philips, The Netherlands). High-resolution 3D T1-weighted turbo field echo magnetization-prepared rapid gradient-echo (MPRAGE) sequence using an eight-channel head coil (TR/TE $=8.5 / 3.046 \mathrm{~ms}, 1 \mathrm{~mm}^{3}$ isotropic voxel size, slice thickness $=1 \mathrm{~mm}$, 160 slices) and a spin-echo EPI sequence (TR/TE $=2000 / 35 \mathrm{~ms}, 3 \mathrm{~mm}^{3}$ voxel size, slice thickness $=4.8,37 / 38$ slices) were acquired. Images were inspected for artefacts and motion; specifically, functional images were visually inspected and corrected for signal dropout and excessive head movement (translation and rotation; range $<3 \mathrm{~mm}$ or $+/-3^{\circ}$ ).

Functional MRI data were analyzed using SPM12 (http://www.fil.ion.ucl. ac.uk/spm). Images underwent slice-timing to TR/2, realignment to the first volume for motion correction, co-registration of the structural image to the mean of the motion corrected images using a 12-parameter affine transformation. Spatial normalization to $1 \mathrm{~mm}^{3}$ and smoothing $(8 \mathrm{~mm}$ FWHM Gaussian kernel) were performed.

\section{Statistical analyses}

First level $t$-test analyses using six contrasts, were defined for each fMRI scanning session including one contrast to assess the overall effect of the scanning session, two contrasts to investigate main effects of trial type (emotion recognition and emotion inhibition), and three to account for the different emotional valences (neutral, negative and positive). Movement parameters were used as regressors in the first level analysis; times at which questions were answered (onsets) were also accounted for. Second level T-contrasts included baseline comparison between diagnostic groups (i.e., BD-placebo vs. HC-placebo); further comparisons included the interaction between drug treatment, physostigmine or placebo, and diagnostic groups (i.e., BD-physostigmine vs. HC-physostigmine; BDplacebo vs. HC-placebo); and the effect of drug treatment within each diagnostic group (i.e., BD-physostigmine vs. BD-placebo; HC-physostigmine vs. HC-placebo). The investigation of emotion-inhibition trials presenting images of negative valence were prioritized in this study to test our a priori hypothesis that subjects with BD would display reduced capacity for inhibitory regulation of muscarinic-cholinergic neurotransmission upon stimulation of the cholinergic system with physostigmine relative to placebo and control groups. Age and gender were included as covariates in all the second level analyses. The threshold for significance was set as $p_{\text {FWE-corr }}<0.05$ in clusters greater than 10-voxels in extent. The Brodmann Atlas [51] was used to localize the significant areas in Talairach space. Exploratory analyses were carried out post-hoc to investigate the contribution of SNP rs324650 on the main cluster findings. Thus, study participants underwent DNA genotyping from saliva samples and were grouped based on their allele frequency for SNP rs324650 (refer to Supplementary Material).

\section{RESULTS}

\section{Participants clinical and demographic characteristics}

The imaging analysis featured a total of 33 participants with BD type I $(n=29)$ and type II $(n=4)$ and 50 controls aged 18-64; groups were matched for age and gender and education level (Table 1). Mood scores were available and examined for 46 participants with BD type I $(n=41)$ and type II $(n=5)$ aged 20-64, and 55 age and gender-matched controls. A total of 18 participants $(N=13 \mathrm{BD}, N=$ 5 controls) were not included in the imaging analysis relative to the mood analysis. This was due to technical port errors in recording the 
Table 1. Clinical and socio-demographics.

\begin{tabular}{|c|c|c|c|}
\hline & $\begin{array}{l}\text { Healthy } \\
\text { control }\end{array}$ & $\begin{array}{l}\text { Bipolar } \\
\text { disorder }\end{array}$ & $\begin{array}{l}\text { Statistical } \\
\text { comparison }\end{array}$ \\
\hline Number of participants & 50 & 33 & \\
\hline Gender, Male/Female (N) & $23 / 27$ & $18 / 15$ & $\begin{array}{l}\chi^{2}(1)=0.581 \\
p=0.446\end{array}$ \\
\hline Age (Years) & $40.1 \pm 13.6$ & $40.6 \pm 11.5$ & $\begin{array}{l}U= \\
828.5,0.974\end{array}$ \\
\hline Male/Female, mean \pm SD & $\begin{array}{l}41.1 \pm 13.5 / \\
39.3 \pm 14.0\end{array}$ & $\begin{array}{l}36.4 \pm 10.7 / \\
45.4 \pm 10.7\end{array}$ & $\begin{array}{l}\mathrm{F}(3,79)=1.511 \\
p=0.218\end{array}$ \\
\hline $\begin{array}{l}\text { Challenge, Placebo/ } \\
\text { Physostigmine }(\mathrm{N})\end{array}$ & $9 / 41$ & $8 / 25$ & $\begin{array}{l}\chi^{2}(1)=0.476 \\
p=0.490\end{array}$ \\
\hline HDRS, mean \pm SD (score) & $1.0 \pm 1.7$ & $8.1 \pm 7.6$ & $\begin{array}{l}U=1430 \\
p<0.001^{*}\end{array}$ \\
\hline median & 0 & 5 & \\
\hline range & $0-7$ & $0-28$ & \\
\hline HARS, mean \pm SD (score) & $0.7 \pm 1.7$ & $5.8 \pm 6.8$ & $\begin{array}{l}U=1319 \\
p<0.001^{*}\end{array}$ \\
\hline median & 0 & 3 & \\
\hline range & $0-8$ & $0-27$ & \\
\hline YMRS, mean \pm SD (score) & $0.7 \pm 1.5$ & $2.2 \pm 2.5$ & $\begin{array}{l}U=1132.5 \\
p=0.001^{*}\end{array}$ \\
\hline median & 0 & 2 & \\
\hline range & $0-6$ & $0-10$ & \\
\hline Age of onset (years) & - & $24.7 \pm 7.8$ & - \\
\hline \multicolumn{4}{|l|}{ mean $\pm S D$} \\
\hline Illness duration (years) & - & $15.0 \pm 9.2$ & - \\
\hline \multicolumn{4}{|l|}{ mean $\pm S D$} \\
\hline \multicolumn{4}{|l|}{ Level of education } \\
\hline Median (score) & 6 & 5 & $\begin{array}{l}\chi^{2}(5)=10.044 \\
p=0.074\end{array}$ \\
\hline Range & $2-7$ & $2-7$ & \\
\hline $\begin{array}{l}\text { Handedness, right/left- } \\
\text { handed }(\mathrm{N})\end{array}$ & $46 / 4$ & $29 / 4$ & $\begin{array}{l}\chi^{2}(51)=52.6 \\
p=0.413\end{array}$ \\
\hline $\begin{array}{l}\text { Medication class } \\
\text { (Frequency, N) }\end{array}$ & - & 2 & - \\
\hline \multicolumn{4}{|l|}{ No medication } \\
\hline Mood stabilizers & - & & - \\
\hline lithium (0.6-1.2 g/day) & & 3 & \\
\hline sodium valproate & & 9 & \\
\hline lamotrigine & & 7 & \\
\hline combination & & 9 & \\
\hline Antidepressants & - & 9 & - \\
\hline Antipsychotics & - & 25 & - \\
\hline Benzodiazepine & - & 2 & - \\
\hline Other Psychotropics & - & 6 & - \\
\hline
\end{tabular}

Data is reported for the imaging sample. $\chi^{2}=$ Chi-squared test; $t=$ independent sample $T$-test; $U=$ Mann-Whitney $U . n=12$ subjects with $B D$ had a HDRS $>8 ; n=4$ subjects had a diagnosis of BD type Il; age of onset is available for $n=29$ subjects with BD. A total of 18 participants $(N=13$ BD, $N=5$ controls) were not included in the imaging analysis relative to the mood analysis. This was due to technical port errors in recording the fMRI behavioural data and a portion of study participants being removed from the scanner prior to completion of the first and/or second fMRI task due to nausea, one of the known peripheral side effects of physostigmine.

fMRI behavioural data and a portion of study participants being removed from the scanner prior to completion of the first and/or second fMRI task due to nausea, one of the known peripheral side effects of physostigmine. For those who did complete imaging, the majority of BD participants were euthymic at the time of scanning (64\%); $n=12$ subjects displayed mild-to-moderate signs and symptoms (Table 1), and we indicate below that removing these subjects failed to alter the findings. Neither mood, as rated using the
POMS-derived four factors depressed, fatigued, positive and irritable $(\mathrm{F}(4,90)=0.332, p=0.856)$, nor word salience $(\mathrm{F}(3,75)=1.546, p=$ 0.210 ) differed significantly at baseline in the $\mathrm{BD}$ group relative to controls. Following physostigmine, these mood factors and word salience were not significantly altered relative to the placebo group and showed no interaction with diagnosis (POMS: $\mathrm{F}(4,90)=0.850, p$ $=0.497$; word salience: $F(3,75)=1.828, p=0.149$ ). Genotype-based recruitment within this sample was challenging in particular for the minor allele ( $\Pi$ genotype; Supplementary Table 2 ) and is considered hereafter as a post-hoc factor to inform future analyses.

\section{Brain functional activation \& emotion recognition and inhibition}

The cholinergic challenge using physostigmine $(1 \mathrm{mg})$ leads to increased functional activation in the posterior cingulate cortices during the inhibition and recognition of negative emotions and affects behavioural performance in BD (without altering mood) relative to healthy controls. While patients' behavioural performance following physostigmine was overall lower than that of controls, physostigmine normalized processing ability (improved accuracy and reaction time) in the BD group during the inhibition of negative emotions, relative to placebo; this effect was associated with cingulate cortex overactivation in BD. We describe these results in detail below, starting with a baseline assessment of behavioural and imaging data in patients relative to controls, followed by an interaction analysis between drug allocation (physostigmine or placebo) and diagnostic group, and, lastly, physostigmine effects within each diagnostic group. The investigation of emotion-inhibition trials presenting images of negative valence were prioritized in this study to test our a priori hypothesis.

\section{Baseline behavioural and imaging analysis}

At baseline, prior to physostigmine administration, we detected impaired emotion processing in $\mathrm{BD}$ relative to the control group overall $(F(36,210)=1.662, p=0.015)$. In particular, having BD was associated with impaired accuracy $(p=0.012)$ and longer time to response $(p=0.029)$ in recognition but not inhibition $(p=0.164$, $p=0.544$ ) of negative emotions. Recognition or inhibition in the context of positive emotion did not differ significantly between diagnostic groups. Impaired recognition of negative emotion was associated with lower right caudate activation $(T=6.21, p<0.001$; Supplementary Fig. 2) and despite being as accurate as controls on the task when inhibiting a negative emotion, this was associated with lower activation of the left subgenual cingulate cortex (BA24, $T=5.39, p<0.001$; Supplementary Fig. 2).

Interaction between drug treatment and diagnostic group on behavioral and imaging data. Physostigmine impacts emotion processing and cingulate activation differently in bipolar disorder relative to healthy controls: We investigated whether physostigmine/ placebo administration would impact brain functional activation and/ or behavioral performance in BD relative to controls. Overall, in accuracy and response times on recognition and inhibition (Supplementary Table 3 ) we detected an effect of diagnosis $(F(12,66)$ $=3.097, p=0.002)$, no effect of physostigmine $(\mathrm{F}(12,66)=1.178, p=$ $0.317)$, and a significant diagnosis-by-physostigmine interaction ( $F$ $(12,66)=1.931, p=0.046$; Supplementary Table 3$)$. This interaction showed that after physostigmine, BD behavioral performance (accuracy) remained significantly lower than controls during the inhibition, but not recognition ( $p=0.495)$, of negative emotions ( $p=$ 0.027 ; Fig. $2 \mathrm{C}$ ). In contrast, prior to receiving the challenge, at baseline, accuracy did not differ between these groups during the inhibition ( $p=0.224)$ or recognition $(p=0.994)$ of negative emotions. Furthermore, in BD-physostigmine administration increased activation in the bilateral dorsal cingulate cortices (BA31) and supramarginal gyri (BA40) during both recognition $(T=4.21, p<0.001$, Fig. $1 C)$ and inhibition $(T=4.11, p<0.001$; Fig. $2 C$ ) of negative and positive ( $T$ 
EMOTION-RECOGNITION

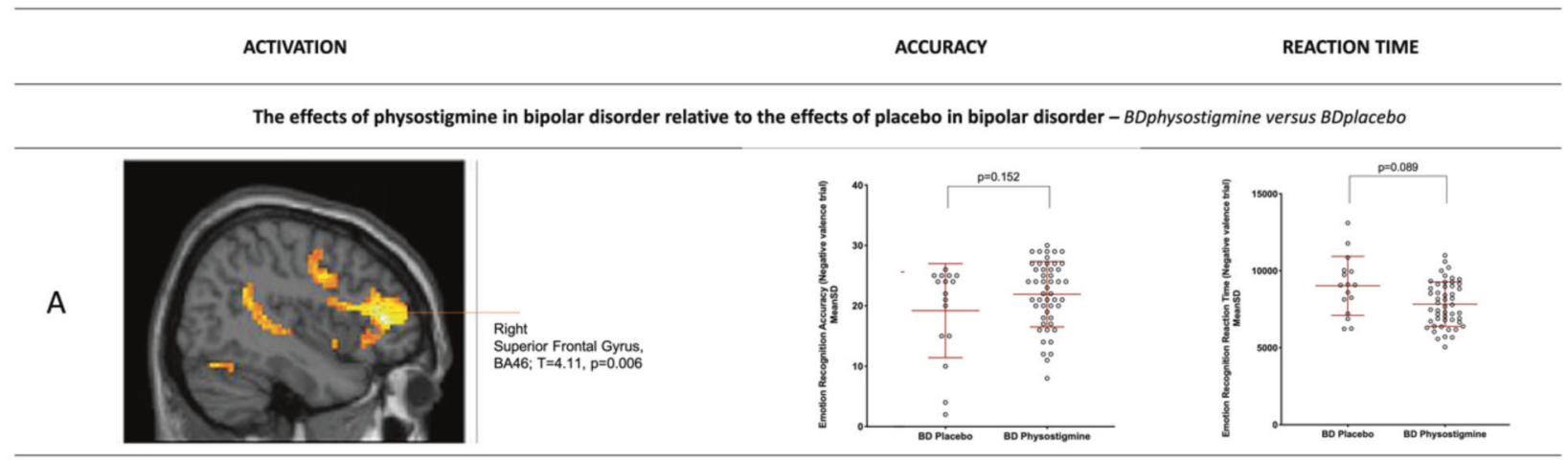

The effects of physostigmine in healthy controls relative to the effects of placebo in healthy controls - HCphysostigmine versus $H C p$ lacebo

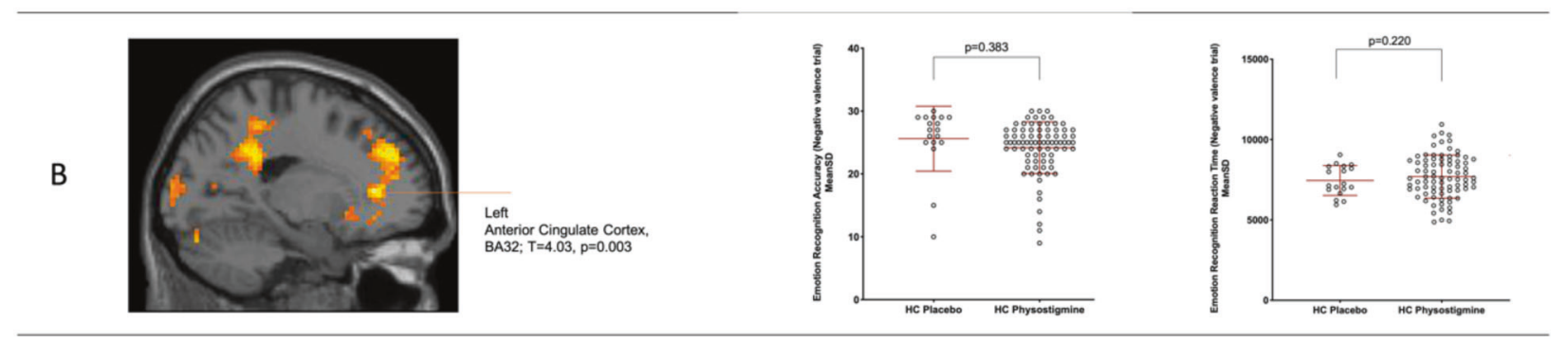

The effects of physostigmine in bipolar disorder relative to the effect of physostigmine in healthy controls - BDphysostigmine versus $H C p h y s o s t i g m i n e$
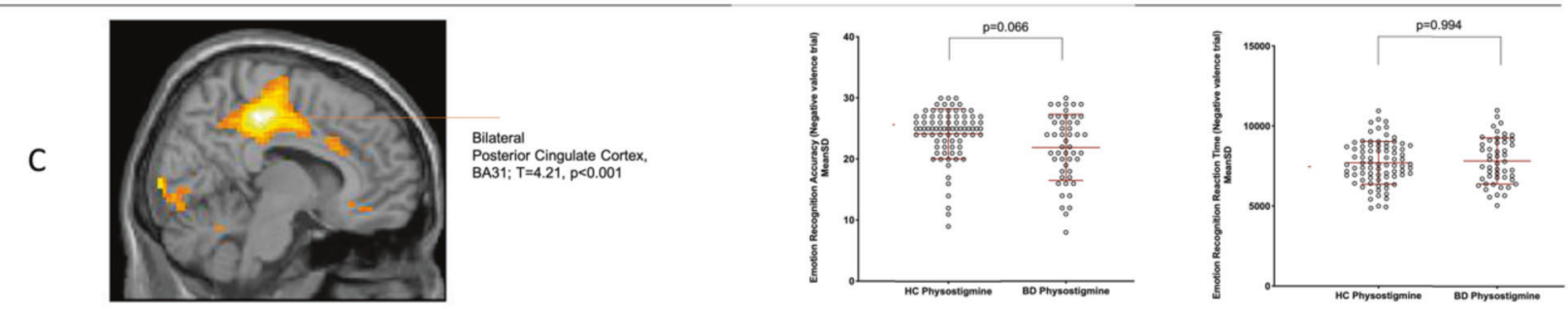

Fig. 1 Physostigmine effects in Bipolar Disorder and Healthy Controls during the recognition of negative emotions. Panel shows the effects of physostigmine on functional activation and behavioural performance during the recognition of negative emotions. $\mathbf{A}$ increased activation in the right superior frontal gyrus (BA46, $T=4.11 p=0.006$ ) in BD receiving physostigmine relative to placebo; $\mathbf{B}$ decreased activation of the left anterior cingulate cortex (BA32, $T=4.03 p=0.003$ ) in controls receiving physostigmine relative to placebo. $\mathrm{C}$ increased activation within the bilateral dorsal cingulate cortices in $\mathrm{BD}$ receiving physostigmine relative to controls (BA31, $T=4.11, p<0.001)$. Behavioural performance (accuracy and reaction times) remained unchanged (A-C). BD Bipolar Disorder; HC Healthy Controls. Phys Physostigmine; ${ }^{*} p_{\text {FWE-corr }}<0.05 ; 10$ voxel-cluster.

$=3.45, p=0.001, T=4.60, p<0.001)$ emotion compared to controls. The main behavioral and imaging findings were confirmed when excluding 9 BD subjects who did not meet criteria for euthymia (refer to Supplementary Material). Post-hoc exploratory analyses including allele frequencies for SNP rs324650 in BD and controls confirmed a statistical effect of physostigmine in the posterior cingulate cortex for the BD-TT group $(n=4)$ on inhibition of negative emotions, with increased activation in the left posterior cingulate cortex (BA23) relative to controls-AA $(n=11$, post-hoc, $p<0.001)$, and bilaterally relative to BD-AT ( $n=11$, post-hoc, BA23; $p=0.0348$ ).

Within-subject comparisons on behavioural and imaging data. Physostigmine effects in healthy controls: Among controls, physostigmine administration relative to placebo did not significantly alter the accuracy or response time in the recognition (Fig. 1B) or inhibition (Fig. 2B) of negative emotions. Despite this, recognition ( $T$ $=4.58, p<0.001$; Fig. 1B) was associated with reduced activation in the left anterior cingulate cortex (BA32), left supramarginal (BA40), right middle occipital gyri (BA19) and right cerebellum, and inhibition ( $T=4.20, p<0.001$; Fig. 2B) with reduced activation of the right caudate and cerebellum; while following physostigmine both recognition and inhibition of negative emotion was associated with reduced activation of the left superior frontal gyrus (BA8).

Physostigmine effects in bipolar disorder: In contrast, among the $B D$ group, physostigmine relative to placebo improved the accuracy and response time on inhibition ( $p=0.071, p=0.007$, Fig. $2 \mathrm{~A}$ ) but not recognition $(p=0.152, p=0.089$, Fig. $1 \mathrm{~A})$ of negative emotion and this was associated with increased activation in the right anterior cingulate (BA32) and middle frontal gyrus (BA10) for inhibition $(T=4.62, p<0.001$; Fig. $2 A)$ and right subgenual cingulate gyrus (BA24) and superior frontal gyrus (BA46) for recognition ( $T=$ 4.11, $p=0.006$; Fig. 1A).

\section{DISCUSSION}

This study demonstrates the involvement of the regulation of the muscarinic-cholinergic neurotransmission in clinically relevant behavioural features of $\mathrm{BD}$, emotion inhibition, relative to a control group and to placebo. At baseline, BD emotion processing was impaired, and their anterior cingulate cortex was under 
EMOTION-INHIBITION

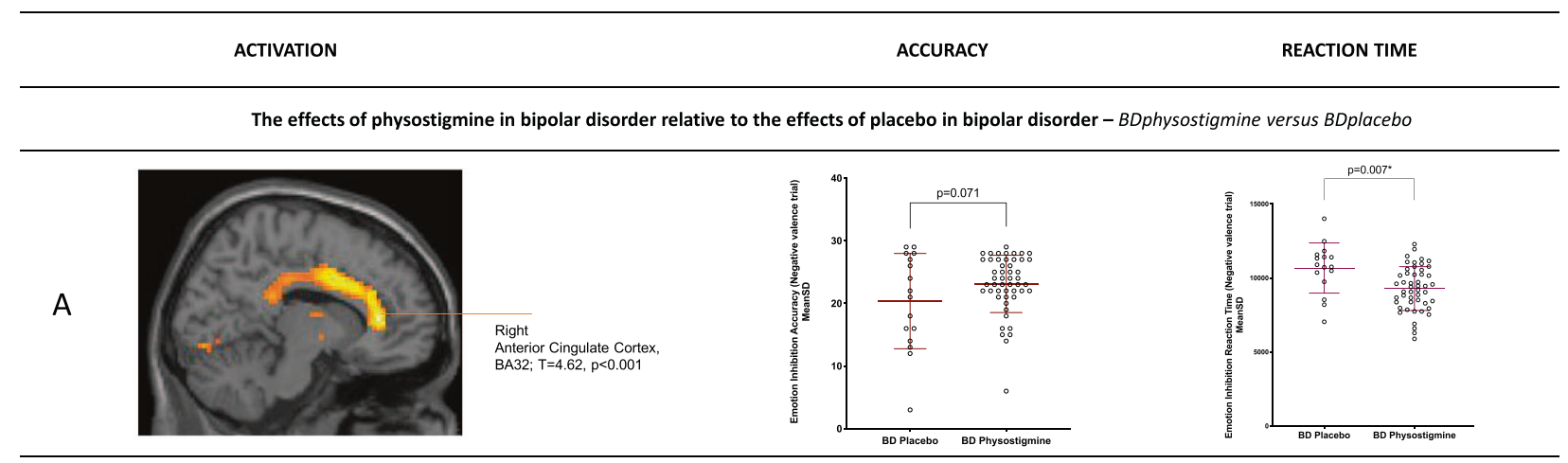

The effects of physostigmine in healthy controls relative to the effects of placebo in healthy controls - HCphysostigmine versus HCplacebo

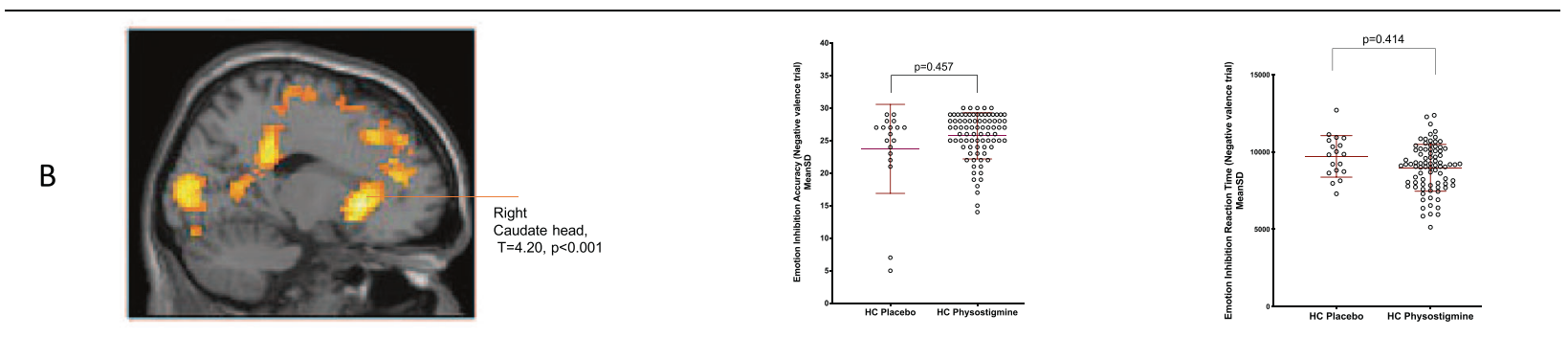

The effects of physostigmine in bipolar disorder relative to the effect of physostigmine in healthy controls - BDphysostigmine versus HCphysostigmine

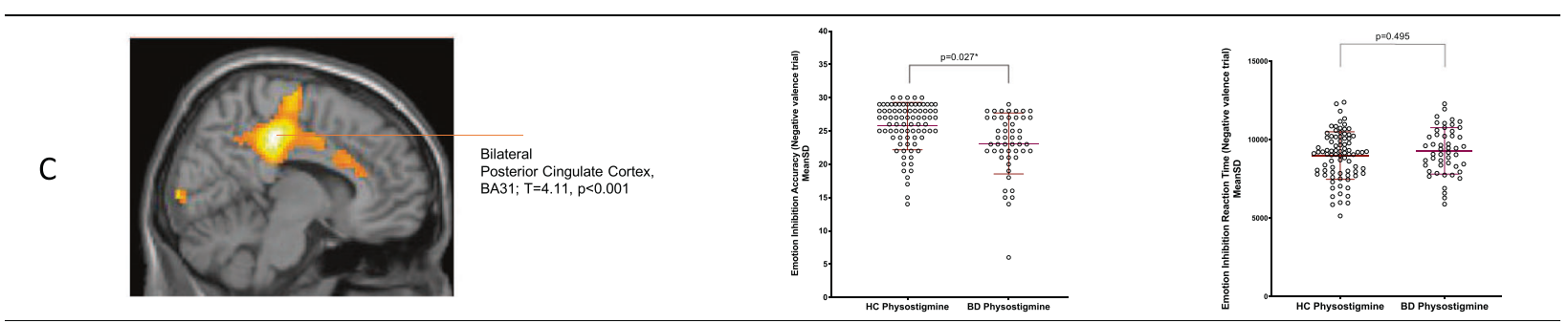

Fig. 2 Physostigmine effects in Bipolar Disorder and Healthy Controls during the inhibition of negative emotions. Panel shows the effects of physostigmine on functional activation and behavioural performance during the inhibition of negative emotions. A increased activation in the right anterior cingulate cortex (BA32, $T=4.62 p<0.001)$ in BD receiving physostigmine relative to placebo; and improved behavioral performance in terms of reaction times $(p=0.007)$; B decreased activation of the right caudate $(T=4.20 p<0.001)$ in controls receiving physostigmine relative to placebo. $C$ increased activation within the bilateral dorsal cingulate cortices (BA31, $T=4.11, p<0.001)$ and improved accuracy in $\mathrm{BD}$ receiving physostigmine relative to placebo $(p=0.027)$, although still statistically lower than that of controls. $B D$ Bipolar Disorder; HC Healthy Controls. Phys Physostigmine; ${ }^{*} p_{\text {FWE-corr }}<0.05 ; 10$ voxel-cluster.

activated, relative to controls. Administration of cholinergic challenge physostigmine affected emotion processing differently in controls than patients, with overactivation observed within the anterior cingulate cortex in $\mathrm{BD}$, and under-activation within the anterior cingulate cortex in controls. Relative to controls, intravenous administration of physostigmine $1 \mathrm{mg}$ at steady state normalized behavioural performance in BD without significantly altering mood (defined by factors depressed, fatigued, irritable, or positive), and was associated with cingulate cortex overactivation in patients, which significantly exceeded that seen in controls (Table 2).

Cannon and collegues [6] previously reported an inverse relationship between the saliency of affective words and wholebrain $M_{2}$ distribution volume in $B D$, in line with the known role of the muscarinic-cholinergic system attributing salience to experiential stimuli [52]. We failed to record group differences in word salience upon muscarinic-cholinergic challenge in BD, possibly due to the lack of effect of physostigmine on mood at the given dosage, which we expected in this sample considering worsening of mood had been observed only at higher dosages in euthymic and actively depressed BD subjects $[15,16]$.

We reported differential behavioural response in $\mathrm{BD}$ relative to controls during the inhibitory regulation or shifting of attention away from emotional stimuli. We also showed that reduced capacity for inhibition of cholinergic neurotransmission in BD was mediated by functional changes within regions involved in ventromedial and ventrolateral routes to emotional and attentional control [1]. The ventromedial route includes the subgenual anterior cingulate cortex, which in task-based fMRI studies of BD has been shown to relate to internal emotion processing such as autobiographical mood induction [1]; and specifically in this study, this may relate to internal processes of emotion inhibition. The ventrolateral route includes the anterior cingulate cortex, which relates to tasks examining external emotion control such as face processing [1], or in this study, image recognition. Anatomically, our imaging findings involve a constellation of functionally specialized subsystems of the brain responsible for the identification of emotionally salient stimuli, the generation of appropriate 
Table 2. Summary of main study findings.

\begin{tabular}{|c|c|c|}
\hline & Behavioral findings & Imaging findings \\
\hline \multicolumn{3}{|l|}{ Baseline } \\
\hline Emotion inhibition & $\downarrow$ Accuracy; $\uparrow$ Reaction time & $\downarrow$ rh Caudate \\
\hline Emotion recognition & - & $\downarrow$ lh Subgenual Cingulate Gyrus (BA24) \\
\hline \multicolumn{3}{|c|}{ Physostigmine effects in BD (vs. Controls) } \\
\hline Emotion inhibition & $\downarrow$ Accuracy & $\begin{array}{l}\uparrow \text { Ih/rh Dorsal Cingulate Cortices (BA31); } \\
\text { Ih/rh Supramarginal Gyri (BA40) }\end{array}$ \\
\hline \multicolumn{3}{|c|}{ Physostigmine effects in HC (vs. placebo) } \\
\hline Emotion inhibition & - & $\downarrow$ Ih Superior Frontal Gyrus (BA8) \\
\hline Emotion recognition & - & $\begin{array}{l}\downarrow \text { Ih Anterior Cingulate Cortex (BA32); } \\
\text { Ih Superior Frontal Gyrus (BA8); } \\
\text { Ih Supramarginal Gyrus (BA40); } \\
\text { rh Middle Occipital Gyrus (BA19); } \\
\text { rh Cerebellum }\end{array}$ \\
\hline Emotion recognition & - & $\begin{array}{l}\uparrow r \text { rh Subgenual Cingulate Gyrus (BA24); } \\
\text { rh Superior Frontal Gyrus (BA46) }\end{array}$ \\
\hline
\end{tabular}

Significant findings from the study are presented for functional activation and behavioural performance for emotion inhibition and recognition trials of negative emotions. BD Bipolar Disorder; HC Healthy Controls; "-" = not significant.

affective state, and the voluntary top-down regulation of emotional responses. Besides the prominent role of the cingulate gyri, other brain areas were recruited. For example, in the BD group at baseline before physostigmine administration, besides the cingulate, the caudate was also under activated when processing emotions relative to controls (Supplementary Fig. 2). The caudate nucleus is one of the areas where emotional states and behaviours originate [32]; when considered with its underactivation during emotion recognition in $\mathrm{BD}$, it suggests patients may not be able to successfully focus on the emotional aspects of a stimulus and that stronger top-down regulation of regions originating emotional response may be needed for attention allocation to emotional stimuli, relative to controls. Following physostigmine in the BD group, in addition to the cingulate cortices (Fig. 2), the superior and middle frontal gyri were overactivated (Fig. 1); these regions collectively participate in ventromedial routes to emotional control and may relate to the deficient regulation of the emotional response elicited by the picture presented, and thus relate to biased valence processing seen in BD. In controls, following physostigmine, besides the cingulate cortices (Fig. 2), parieto-occipital regions were under activated during emotion inhibition, presenting an extensive inhibitory neurocircuitry functional model associated with emotion processing in healthy controls.

In summary, in controls, physostigmine induced reductions in cingulate functional activity, while emotion processing remained broadly unchanged, may reflect an intact autoreceptor system response to cholinergic challenge in this group. Contrastingly in $B D$, physostigmine induced increased activation within the cingulate cortex suggests a molecular deficit in this group, in line with the proposed reductions in inhibitory capacity seen in BD [29]. Of note, Cannon and colleagues [29] reported that it was not only in the cingulate cortex that the $M_{2}$ receptors were reduced in $\mathrm{BD}$; however, the ACC was the region displaying the statistically strongest differences [29]. While the cingulate cortex role within networks involved in emotion processing deficits is well established in BD both anatomically and functionally [1, 53], the neurotransmitter systems involved in these impairments, and more generally the contribution of these systems to mood dysregulation and cognitive control in psychiatry, are less investigated.

A preliminary look into the role of the rs324650 SNP in muscarinic-cholinergic function in BD following physostigmine administration showed greater activation in the posterior cingulate cortices during the inhibition of negative emotions in the BD-TT genotype group, relative to BD-AT and controls-AA; in line with reduced capacity for inhibition of cholinergic neurotransmission seen overall in BD in this study. Cannon and colleagues [30] previously showed that the $\Pi$ polymorphism in BD was linked with a higher risk of attempting suicide, poorer social-occupational function and greater cognitive impairment. Future studies including further clinical and behavioural variables should investigate whether these deficits may reflect poorer cognitive reserve for BD subjects who are homozygous for the $T$ allele. The effect of genetic variance in rs324650 on $\mathrm{M}_{2}$-receptor binding in the cingulate cortices in $\mathrm{BD}$ has been previously described [30], with BD-TT genotype showing the most significant reductions in $\mathrm{M}_{2}$-receptor binding (lower $\mathrm{M}_{2}$-receptor distribution volume) relative to BD-AT and controls-TT. Considering this receptor plays a major role in the regulation of $\mathrm{ACh}$ release, a genetic variation within $\mathrm{CHRM} 2$ that alters the function of this autoreceptor could, in turn, alter the capacity for regulation of cholinergic neurotransmission and thus exert widespread effects on a variety of emotional and cognitive domains and their interaction, particularly in $\mathrm{BD}$.

It is to be noted that our BD sample was taking a range of medications at the time of scanning. Their effect and interaction on physostigmine were beyond the scope of this study. Although we cannot exclude medication effects on our findings, a recent review of medication effects in neuroimaging studies in BD suggests that medication impact is higher for structural (volumetric) MRI than it is for diffusion or functional MRI [54].

In closing, physostigmine induced increase in cingulate cortex functional activation during emotion processing in BD may be considered a marker of cholinergic sensitivity and emotion dysregulation in this illness. When considered together with the 
normalization of emotion processing ability seen in the BD group in this study, these physostigmine induced effects may represent a valid dose-sensitive target for core symptom management in BD. Recently, the relative increase in acetylcholine concentrations and cholinergic signalling dysregulation in $\mathrm{BD}$ has been a potential target for muscarinic antagonists such as scopolamine [55], which has been proven a fast-onset and sustained antidepressant in medication naïve and treatment-resistant BD [56]; however, its dose-response profile and time-course antidepressant effects and impact on cognition will require further investigation. We also report preliminary evidence of cingulate involvement in a subgroup of $B D$ participants with the $\Pi$ genotype of SNP rs324650. While preliminary evidence suggests we may be coming closer to being able to exploit the cholinergic hypothesis of mood $[16,55]$ to ameliorate core features of BD illness, further clinical trials using muscarinic-cholinergic antagonists are warranted and future research should strive to incorporate a genomics approach to define muscarinic-cholinergic sensitivity to potentially detect those individuals more likely to benefit from these agents.

\section{REFERENCES}

1. Strakowski SM, Adler CM, Almeida J, Altshuler LL, Blumberg HP, Chang KD, et al. The functional neuroanatomy of bipolar disorder: A consensus model. Bipolar Disord. 2012;14:313-25.

2. Krahn GL. WHO World report on disability: a review. Disabil Health J. 2011;4:141-2.

3. Yildiz A, Vieta E, Leucht S, Baldessarini RJ. Efficacy of antimanic treatments: Metaanalysis of randomized, controlled trials. Neuropsychopharmacology. 2011;36: 375-89.

4. Townsend J, Altshuler LL. Emotion processing and regulation in bipolar disorder: A review. Bipolar Disord. 2012;14:326-39.

5. Bell CC. DSM-IV: diagnostic and statistical manual of mental disorders. JAMA. 1994:272:828-9.

6. Cannon DM, Nugent AC, Erickson K, Carson RE, Eckelman WC, Kiesewetter DO, et al. Muscarinic cholinergic2 receptor binding in bipolar disorder: Relation to saliency of affective-words. J Cereb Blood Flow Metab. 2005;25:S420-S420.

7. Hummer TA, Hulvershorn LA, Karne HS, Gunn AD, Wang Y, Anand A. Emotional response inhibition in bipolar disorder: $A$ functional magnetic resonance imaging study of trait- and state-related abnormalities. Biol Psychiatry. 2013;73:136-43.

8. Shine JM, Breakspear M, Bell PT, Ehgoetz Martens KA, Shine R, Koyejo O, et al. Human cognition involves the dynamic integration of neural activity and neuromodulatory systems. Nat Neurosci. 2019;22:289-96.

9. Janowsky DS, Davis JM, El-Yousef MK, Sekerke HJ. A cholinergic-adrenergic hypothesis of mania and depression. Lancet. 1972;300:632-5.

10. van Enkhuizen J, Janowsky DS, Olivier B, Minassian A, Perry W, Young JW, et al. The catecholaminergic-cholinergic balance hypothesis of bipolar disorder revisited. Eur J Pharmacol. 2015;753:114-26.

11. Furey ML, Pietrini P, Haxby JV, Drevets WC. Selective effects of cholinergic modulation on task performance during selective attention. Neuropsychopharmacology. 2008;33:913-23.

12. Bentley $P$, Vuilleumier $P$, Thiel $C M$, Driver J, Dolan RJ. Cholinergic enhancement modulates neural correlates of selective attention and emotional processing. Neuroimage. 2003;20:58-70.

13. Bentley $P$, Vuilleumier $P$, Thiel CM, Driver J, Dolan RJ. Effects of attention and emotion on repetition priming and their modulation by cholinergic enhancement. J Neurophysiology. 2019;90:1171-81.

14. Je Jeon W, Dean B, Scarr E, Gibbons A. The role of muscarinic receptors in the pathophysiology of mood disorders: a potential noveltreatment? Curr Neuropharmacol. 2015;13:739-49.

15. Modestin, J, Hunger, J, Schwartz, R. Uber die depressogene Wirkung yon Physostigmin. Archiv für Psychiatrie und Nervenkrankheiten. 1973;218:67-77.

16. Janowsky DS, Khaled El Yousef M, Davis JM. Acetylcholine and depression. Psychosom Med. 1974;36:248-57.

17. Risch SC, Cohen RM, Janowsky DS, Kalin NH, Sitaram N, Gillin JC, et al. Physostigmine induction of depressive symptomatology in normal human subjects. Psychiatry Res. 1981;4:89-94.

18. KL Davis, LE Hollister, J Overall, A Johnson, K. Train psycho pharmacology physostigmine: effects on cognition and affect in normal subjects. Psychopharmacology. 1976;27:23-27.

19. Janowsky DS, Risch SC, Kennedy B, Ziegler M, Huey L. Central muscarinic effects of physostigmine on mood, cardiovascular function, pituitary and adrenal neuroendocrine release. Psychopharmacol. 1986;89:150-4.
20. Risch SC, Janowsky DS, Mott MA, Gillin JC, Kalir HH, Huey LY, et al. Central and peripheral cholinesterase inhibition: effects on anterior pituitary and sympathomimetic function. Psychoneuroendocrinology. 1986;11:221-30.

21. Carroll BJ, Greden JF, Haskett R, Feinberg M, Albala AA, Martin FI, et al. Neurotransmitter studies of neuroendocrine pathology in depression. Acta Psychiatr Scand Suppl. 1980;280:183-99.

22. Fleischhacker WW, Barnas C, Günther V, Meise U, Stuppäck C, Unterweger B. Mood-altering effects of biperiden in healthy volunteers. J Affect Disord. 1987;12:153-7.

23. Carroll BJ, Frazer A, Schless A, Mendels, J. Cholinergic reversal of manic symptoms. Lancet. 1972;299:427-8.

24. Kenneth LD, Berger PA, Hollister LE, Defraites E. Physostigmine Cholinergic. Arch Gen Psychiatry. 1978;35:119-22.

25. Shopsin B, Janowsky D, Davis J, Gershon S. Rebound phenomena in manic patients following physostigmine. Neuropsychobiology. 1975;1:180-7.

26. Janowsky DS, El-Yousef MK, Davis JM, Sekerke HJ. Parasympathetic suppression of manic symptoms by physostigmine. Arch Gen Psychiatry. 1973;28:542-7.

27. Janowsky DS, El-yousefjohn K, Dais M, Hubbard B, Sekerke HJ. Cholinergic reversal of manic symptoms. Lancet. 1971;299:1236-7.

28. Scarr E. Muscarinic receptors: their roles in disorders of the central nervous system and potential as therapeutic targets. CNS Neurosci Ther 2012; 18:369-79.

29. Cannon DM, Carson RE, Nugent AC, Eckelman WC, Kiesewetter DO, Williams J, et al. Reduced muscarinic type 2 receptor binding in subjects with bipolar disorder. Arch Gen Psychiatry. 2006;63:741-7.

30. Cannon DM, Klaver JK, Gandhi SK, Solorio G, Peck SA, Erickson K, et al. Genetic variation in cholinergic muscarinic-2 receptor gene modulates $M 2$ receptor binding in vivo and accounts for reduced binding in bipolar disorder. Mol Psychiatry. 2010;16:407-18.

31. Gosso FM, de Geus EJ, Polderman TJ, Boomsma DI, Posthuma D, Heutink P. Exploring the functional role of the CHRM2 gene in human cognition: Results from a dense genotyping and brain expression study. BMC Med Genet. 2007;8:1-12.

32. Phillips ML, Ladouceur CD \& Drevets WC. A neural model of voluntary and automatic emotion regulation: implications for understanding the pathophysiology and neurodevelopment of bipolar disorder. 833-57.2008. https://doi.org/ 10.1038/mp.2008.65.

33. Caseras X, Murphy K, Lawrence NS, Fuentes-Claramonte P, Watts J, Jones DK, et al. Emotion regulation deficits in euthymic bipolar I versus bipolar II disorder: A functional and diffusion-tensor imaging study. Bipolar Disord. 2015;17:461-70.

34. Chase HW \& Phillips ML. Elucidating neural network functional connectivity abnormalities in bipolar disorder: toward a harmonized methodological approach. 1-22. 2017. https://doi.org/10.1016/j.bpsc.2015.12.006.Elucidating.

35. Strakowski SM, DelBello MP, Adler CM. The functional neuroanatomy of bipolar disorder: a review of neuroimaging findings. Mol Psychiatry. 2005;10:105-16.

36. Townsend JD, Bookheimer SY, Foland-Ross LC, Moody TD, Eisenberger NI, Fischer JS, et al. Deficits in inferior frontal cortex activation in euthymic bipolar disorder patients during a response inhibition task. Bipolar Disord. 2012;14:442-50.

37. Malhi G, Lagopoulos J, Sachdev P, Ivanovski B \& Shnier R. An emotional Stroop functional MRI study of euthymic bipolar disorder. Bipolar Disord. 2005;7:58-69.

38. Rey G, Desseilles M, Favre S, Dayer A, Piguet C, Aubry JM, et al. Modulation of brain response to emotional conflict as a function of current mood in bipolar disorder: preliminary findings from a follow-up state-based fMRI study. Psychiatry Res Neuroimaging. 2014;223:84-93.

39. Elliott R, Ogilvie A, Rubinsztein JS, Calderon G, Dolan RJ, Sahakian BJ. Performance of an affective go/no go task in patients with mania. Biol Psychiatry. 2004;55:1163-70. https://doi.org/10.1016/j.biopsych.2004.03.007.

40. Phillips ML, Drevets WC, Rauch SL, Lane R. Neurobiology of emotion perception II: Implications for major psychiatric disorders. Biol Psychiatry. 2003;54:515-28.

41. Ochsner KN, Gross JJ. The cognitive control of emotion. Trends Cogn Sci. 2005;9:242-9.

42. Mesulam M. Central cholinergic pathways neuroanatomy and some behavioral implications. In Avoli M, Reader TA, Dykes RW \& Gloor P, editors. Neurotransmitters and cortical function: from molecules to mind. Springer US: 1988. p. 237-60. https://doi.org/10.1007/978-1-4613-0925-3_15.

43. Furey ML, Pietrini P, Haxby JV, Alexander GE, Lee HC, VanMeter J, et al. Cholinergic stimulation alters performance and task-specific regional cerebral blood flow during working memory. Proc Natl Acad Sci. 2002;94:6512-6.

44. Oduro KA. Glycopyrrolate methobromide 2. Comparison with atropine sulphate in anaesthesia. Can Anaesth Soc J. 1975;22:466-73.

45. American Psychiatric Association. Diagnostic and statistical manual of mental disorders. Washington, DC; 2013.

46. Luria RE. The validity and reliability of the visual analogue mood scale. J Psychiatr Res. 1975;12:51-57. 
47. FC Murphy, BJ Sahakian, JS Rubinsztein, A Michael, RD Rogers, TW Robbins, et al. Emotional bias and inhibitory control processes in mania and depression. Psychological Med.1999;29:1307-21.

48. Tozzi L, Doolin K, Farrel C, Joseph S, O'Keane V, Frodl T. Functional magnetic resonance imaging correlates of emotion recognition and voluntary attentional regulation in depression: A generalized psycho-physiological interaction study. J Affect Disord. 2017;208:535-44.

49. Lisiecka DM, Carballedo A, Fagan AJ, Connolly G, Meaney J, Frodl T. Altered inhibition of negative emotions in subjects at family risk of major depressive disorder. J Psychiatr Res. 2012;46:181-8.

50. J Lang, P, M Bradley, M. \& N \& Cuthbert, B. International Affective Picture System (IAPS): Affective Ratings of Pictures and Instruction Manual (Rep. No. A-8). Technical Report A-8 (2008).

51. Brodmann K. Brodmann's Localisation in the Cerebral Cortex. World Scientific. 1909

52. McGaugh JL. The amygdala modulates the consolidation of memories of emotionally arousing experiences. Annu Rev Neurosci. 2004;27:1-28.

53. Perry A, Roberts G, Mitchell PB \& Breakspear M. Connectomics of bipolar disorder: a critical review, and evidence for dynamic instabilities within interoceptive networks. Mol. Psychiatry. 2018. https://doi.org/10.1038/s41380-018-0267-2.

54. Hafeman D, Chang K, Garrett A, Sanders E \& Phillips M. Effects of medication on neuroimaging findings in bipolar disorder: an updated review. Bipolar Disord. 375-410.2012. https://doi.org/10.1111/j.1399-5618.2012.01023.x.

55. Dulawa SC \& Janowsky DS. Cholinergic regulation of mood: from basic and clinical studies to emerging therapeutics. Mol Psychiatry. 2018. https://doi.org/ 10.1038/s41380-018-0219-x.

56. Ellis JS, Zarate CA, Luckenbaugh DA, Furey ML. Antidepressant treatment history as a predictor of response to scopolamine: clinical implications. J Affect Disord. 2014:162:39-42.

\section{ACKNOWLEDGEMENTS}

We would like to gratefully acknowledge the participants for taking part in this research study. This work is dedicated to the memory of Dorothy May Reynolds Cannon.

\section{AUTHOR CONTRIBUTIONS}

LN: recruitment, analysis, writing; JF: data analysis; GMcP: recruitment; LK: data analysis; MRD: data analysis; TNA: data analysis; PN: recruitment; SA: recruitment and analysis; FMM: recruitment; JMCL: recruitment; MG: recruitment; JM: recruitment; DM: data analysis; TF: data analysis; CMCD: recruitment and editing; $\mathrm{BH}$ : recruitment and editing; DMC: design, funding, supervision of analysis and writing.

\section{FUNDING}

This research is supported by the Irish Research Council (IRC) Postgraduate Scholarship, Ireland awarded to Leila Nabulsi, PhD, and by the Health Research Board (HRA-POR-324) awarded to Dr Dara M. Cannon, PhD. We gratefully acknowledge the participants and the support of the Wellcome-Trust HRB Clinical Research Facility and the Centre for Advanced Medical Imaging, St. James Hospital, Dublin, Ireland. Open Access funding provided by the IReL Consortium.

\section{COMPETING INTERESTS}

The authors declare no competing interests.

\section{ADDITIONAL INFORMATION}

Supplementary information The online version contains supplementary material available at https://doi.org/10.1038/s41386-022-01268-7.

Correspondence and requests for materials should be addressed to Leila Nabulsi.

Reprints and permission information is available at http://www.nature.com/ reprints

Publisher's note Springer Nature remains neutral with regard to jurisdictional claims in published maps and institutional affiliations.

(i) Open Access This article is licensed under a Creative Commons Attribution 4.0 International License, which permits use, sharing, adaptation, distribution and reproduction in any medium or format, as long as you give appropriate credit to the original author(s) and the source, provide a link to the Creative Commons license, and indicate if changes were made. The images or other third party material in this article are included in the article's Creative Commons license, unless indicated otherwise in a credit line to the material. If material is not included in the article's Creative Commons license and your intended use is not permitted by statutory regulation or exceeds the permitted use, you will need to obtain permission directly from the copyright holder. To view a copy of this license, visit http://creativecommons. org/licenses/by/4.0/.

(c) The Author(s) 2022 